\title{
Direct measurement of group dispersion of optical components using white-light spectral interferometry
}

\author{
R. CHLEBUS, P. HLUBINA*, and D. CIPRIAN \\ Department of Physics, Technical University of Ostrava, \\ 17. listopadu 15, 708-33 Ostrava-Poruba, Czech Republic
}

\begin{abstract}
We present a simple white-light spectral interferometric technique employing a low-resolution spectrometer for a direct measurement of the group dispersion of optical components over a wide wavelength range. The technique utilizes an unbalanced Mach-Zehnder interferometer with a component under test inserted in one arm and the other arm with adjustable path length. We record a series of spectral interferograms to measure the equalization wavelength as a function of the path length difference. We measure the absolute group refractive index as a function of wavelength for a quartz crystal of known thickness and the relative one for optical fiber. In the latter case we use a microscope objective in front and a lens behind the fiber and subtract their group dispersion, which is measured by a technique of tandem interferometry including also a Michelson interferometer.
\end{abstract}

Keywords: spectral interferometry, white-light source, low-resolution spectrometer, Mach-Zehnder interferometer, group refractive index, dispersion, quartz crystal, holey fiber.

\section{Introduction}

The group dispersion, that is, the wavelength dependence of the group refractive index, belongs to one of the fundamental dispersion characteristics of both isotropic and anisotropic optical materials. White-light interferometry based on the use of a white-light source in combination with a standard Michelson or Mach-Zehnder interferometer, is considered as one of the best tools to measure the group dispersion for different optical materials over a broad spectral range.

White-light interferometry usually utilizes either of two methods, that is, a temporal method or a spectral method, depending on whether interference is observed in the time domain or in the spectral domain, respectively. The temporal method involves measurement of the time of flight of optical pulses through a sample. A method for measuring the group delay introduced by an optical material consists in placing the sample in one arm of the interferometer and evaluating the temporal shift of the peak of the cross-correlation interferogram. As the central wavelength is varied, the relative group delay of the different frequency components is observed directly [1]. Alternatively, the spectral phase over the full bandwidth of the white-light source can be obtained in a single measurement by a Fourier transform of the cross-correlation interferogram [2,3]. The dispersion characteristics of the sample under study can be obtained by simply differentiating the measured spectral phase.

\footnotetext{
*e-mail: petr.hlubina@vsb.cz
}

The spectral method is based on the observation of channelled spectrum [4-6] and involves measurement of the period of the spectral fringes in the vicinity of a stationary-phase point [4,7] that appears in the recorded spectral interferogram when the group optical path difference (OPD) between two beams in the interferometer is close to zero. The main limitation of the method is reached for thick or strongly dispersive materials because under such conditions the spectral interference fringes that are far from the stationary-phase point become difficult to resolve. Fortunately, the measurement of the group refractive index dispersion of a given material is still possible in the vicinity of the stationary-phase point if one moves it in successive steps to different wavelengths [8] and repeats the measurement. The modification of the technique with a tandem configuration of a Michelson interferometer and a calcite crystal of known thickness has been used in measurement of the group birefringence dispersion [9].

In this paper, a simple white-light spectral interferometric technique employing a low-resolution spectrometer is used for a direct measurement of the group dispersion of optical components over a wide wavelength range. An unbalanced Mach-Zehnder interferometer with a component under test inserted in one arm and the other arm with adjustable path length is used and a series of spectral interferograms is recorded to measure the equalization wavelength as a function of the path length difference. We measured by the technique the group refractive index as a function of wavelength for a quartz crystal of known thickness. Also the relative group refractive index dispersion for opti- 
cal fiber was measured. In this case we used a microscope objective in front and a lens behind the fiber and subtracted their group dispersion, which was measured by a technique of tandem interferometry employing also a Michelson interferometer.

\section{Experimental method}

Let us consider a sample under test of the thickness $d$ and the refractive index $n(\lambda)$ inserted into the first (test) arm of an unbalanced Mach-Zehnder interferometer as shown in Fig. 1. If the other arm is with the adjustable path length $L$ in the air, the group OPD $\Delta_{\mathrm{MZ}}^{\mathrm{g}}(\lambda)$ between the beams in the interferometer is given by

$$
\Delta_{\mathrm{MZ}}^{\mathrm{g}}(\lambda)=(L-l)-d[N(\lambda)-1],
$$

where $l$ is the path length in the air in the test arm prior to inserting the sample in the interferometer and $N(\lambda)=$ $n(\lambda)-\lambda \mathrm{d} n(\lambda) / \mathrm{d}(\lambda)$ is the group refractive index.

When the case of thick or strongly dispersive materials is considered, the spectral interference fringes recorded in the setup have the largest period in the vicinity of a stationary-phase point for which the group OPD is zero at one specific wavelength $\lambda_{0}$, the so-called equalization wavelength [8], satisfying the relation $\Delta_{\mathrm{MZ}}^{\mathrm{g}}\left(\lambda_{0}\right)=0$. It gives, for the path length $L=L\left(\lambda_{0}\right)$ for which the equalization wavelength $\lambda_{0}$ is resolved in the recorded spectrum, the relation

$$
L\left(\lambda_{0}\right)=l+d\left[N\left(\lambda_{0}\right)-1\right],
$$

If we introduce the path length difference $\Delta L\left(\lambda_{0}\right)=$ $L\left(\lambda_{0}\right)-L_{0}$ where $L=L_{0}=l$ is the path length for the balanced interferometer (without the sample and with the zero OPD), we obtain the simple relation

$$
N\left(\lambda_{0}\right)=1+\Delta L\left(\lambda_{0}\right) / d,
$$

enabling to measure directly the group refractive index $N\left(\lambda_{0}\right)$ as a function of the equalization wavelength $\lambda_{0}$ for a sample of the known thickness $d$. If one of the equalization wavelengths is chosen as the reference one $\lambda_{0 r}$, we can measure the relative or differential group refractive index $\Delta N\left(\lambda_{0}\right)=N\left(\lambda_{0}\right)-N\left(\lambda_{0 r}\right)$.

We can estimate the minimum and maximum thicknesses of the sample whose group dispersion can be measured by the method. If the measurement is restricted to the wavelength range from $\lambda_{0 \min }$ to $\lambda_{0 \max }$ and we assume normal dispersion and no limitation due to the resolving power of the spectrometer, the minimum and maximum thicknesses, $d_{\min }$ and $d_{\max }$, are given by the minimum and maximum path length differences, $\Delta L_{\min }$ and $\Delta L_{\max }$, adjustable in the interferometer

$$
d_{\text {min,max }}=\Delta L_{\min , \max } /\left[N\left(\lambda_{0 \max , \min }\right)-1\right] .
$$

The group dispersion of samples with the thicknesses below $d_{\min }$ can be measured by a method of tandem interferometry based on the fact that the group OPD in the interferometer with the sample is compensated by the OPD adjusted in the other interferometer such as a Michelson one.

\section{Experimental setup}

The experimental setup used in the application of spectral-domain white-light interferometry to measure the group dispersion of a sample under test is shown in Fig. 1. It consists of a white-light source: a quartz-tungsten-halogen lamp (HL-2000HP, Ocean Optics, Inc.) with launching optics, optical fiber of cutoff wavelength as short as possible, a collimating lens, a bulk-optic Mach-Zehnder interferometer with plate beam splitters (BSW07, Thorlabs), a

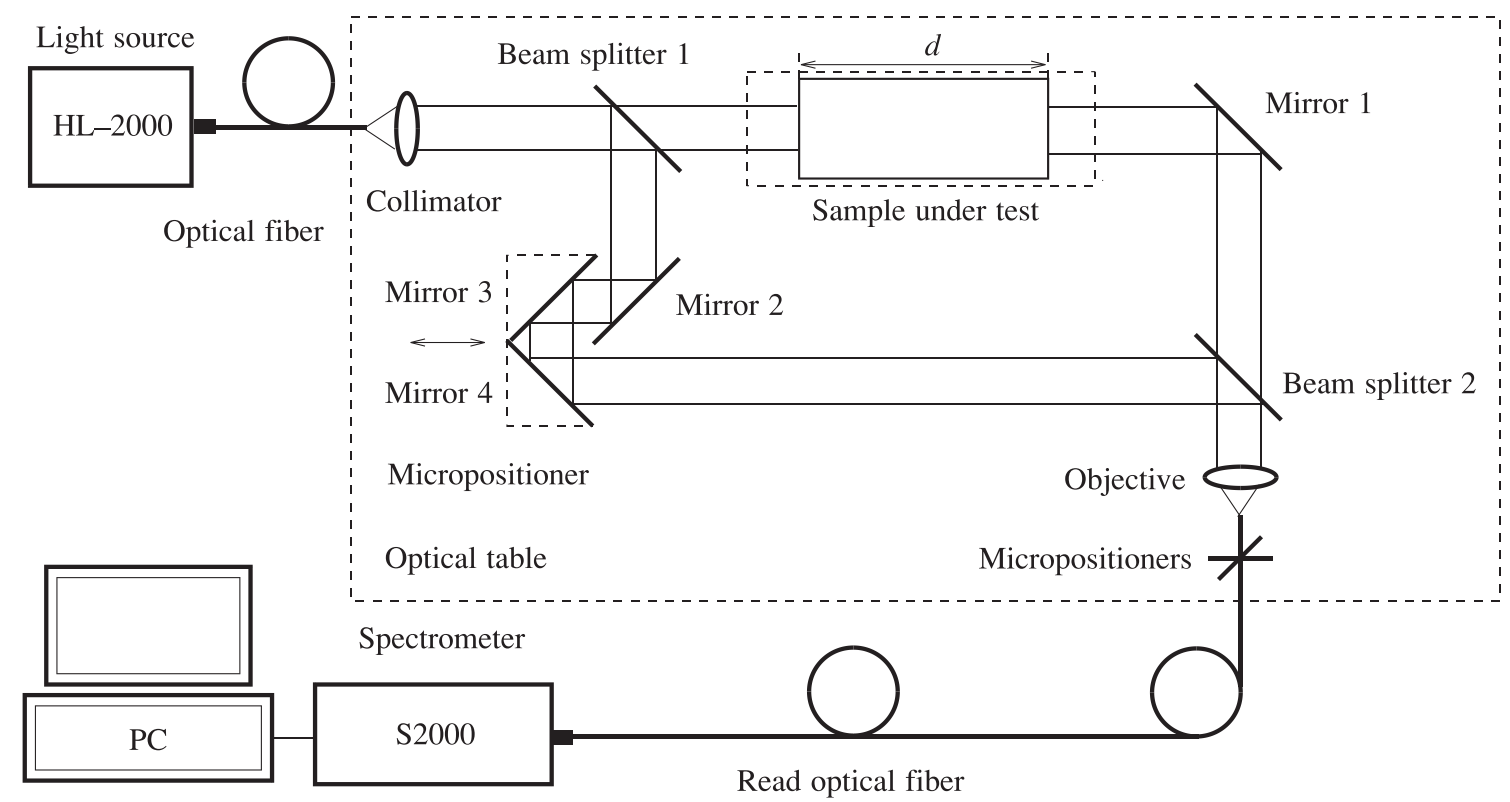

Fig. 1. Experimental setup with a low-resolution spectrometer and an unbalanced Mach-Zehnder interferometer to measure the group dispersion in a sample under test. 


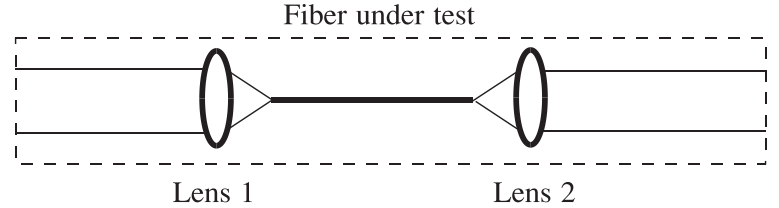

(a)

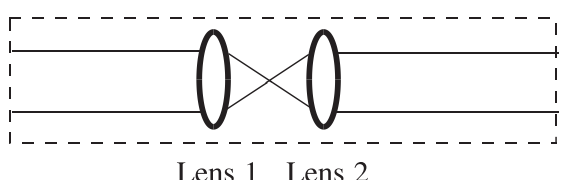

Lens 1 Lens 2

(b)

Fig. 2. Second combination of components: two lenses and fiber under test (a), third combination of components: two lenses (b).

micropositioner connected to mirrors 3 and 4 of the interferometer, a microscope objective, micropositioners, a fiber-optic spectrometer (S2000, Ocean Optics, Inc.), an A/D converter and a personal computer. The sample under test is quartz crystal that consists of two polished surfaces, perpendicular to the optic axis of the crystal with a precision of 15 arcmin. The crystal is placed into the test arm of the interferometer in such a way that the collimated beam is incident on the surfaces perpendicularly as shown in Fig. 1. The thickness of the crystal is $d=4089 \pm 10 \mu \mathrm{m}$.

In second arrangement, the sample under test is replaced by a combination of components shown schematically in Fig. 2(a). They are represented by a microscope objective $(10 \times / 0.30$, Meopta), a fused-silica holey fiber (PM-1550-01, Thorlabs) of the length $z=(50650 \pm 10) \mu \mathrm{m}$, and an achromatic lens (74-ACR, Ocean Optics, Inc.). In the last arrangement, we used a combination of two components not including the fiber as shown schematically in Fig. 2(b).

\section{Experimental results and discussion}

First, we determined the virtual (not adjustable) path length $L_{0}$ corresponding to the balanced Mach-Zehnder interferometer. We used a method of tandem interferometry and placed a Michelson interferometer in between the source and the unbalanced Mach-Zehnder interferometer with the adjusted path length $L$. We used the fact that the spectral interference fringes are observable for the zero OPD in the

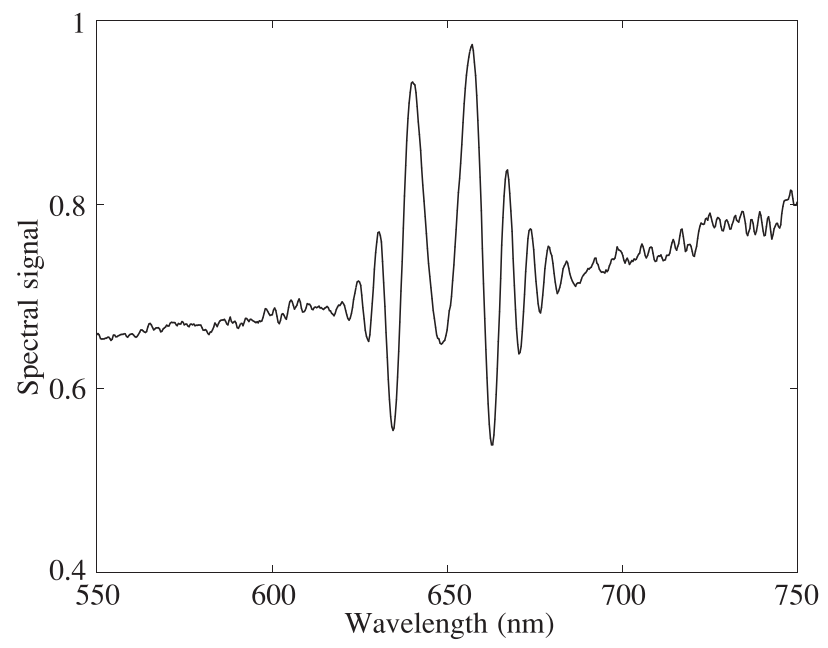

Fig. 3. Example of the spectral signal recorded for the path length difference $\Delta L=23000 \mu \mathrm{m}$ (quartz crystal).
Michelson interferometer and for such an OPD which is the same as the OPD adjusted in the unbalanced MachZehnder interferometer. The OPD is equal to the path length difference $\Delta L=L-L_{0}$, which was determined in this way with a precision better then $1 \mu \mathrm{m}$. Then we measured the group dispersion of a quartz crystal, parameters of which are presented above, in the setup shown in Fig. 1. The main advantage of the setup is in fiber connection of a light source (that can be varied) with the interferometer. Using a laser diode instead of the halogen lamp, we checked precise placement of the crystal sample in the test arm by observing the interference fringes.

The group dispersion of the quartz crystal was measured by adjusting such a path length to resolve spectral interference fringes. Figure 3 shows an example of the spectral signal recorded for the path length difference $\Delta L=23000 \mu \mathrm{m}$. In the spectral signal it is clearly seen the effect of the limiting resolving power of the spectrometer on the visibility of the spectral interference fringes identified only in the vicinity of the equalization wavelength $\lambda_{0}=648.23 \mathrm{~nm}$. The equalization wavelength was determined by an autoconvolution method [10] with an error of $0.32 \mathrm{~nm}$ corresponding to the wavelength difference for adjacent pixels.

We measured in this way the dependence of the adjusted path length difference on the equalization wavelength. We displaced the stage with mirrors 3 and 4 manually by using the micropositioner with a constant step of $10 \mu \mathrm{m}$ and performed recording of the corresponding spectral signals. The recorded spectral signals revealed that the equalization wavelength $\lambda_{0}$ can be resolved in the spectral range from 490 to $899 \mathrm{~nm}$ and that the path length difference $\Delta L$ varies from 23820 to $22540 \mu \mathrm{m}$. Knowledge of the measured dependence and the sample thickness $d$ enabled us to evaluate directly the group refractive index $N\left(\lambda_{0}\right)$ of the quartz crystal as a function of the equalization wavelength $\lambda_{0}$. The function is represented in Fig. 4 by the dots and it is shown together with the theoretical function resulting from the dispersion relation proposed by Ghosh [11]. We can estimate a precision of the group refractive index measurements [12]. If the path length difference $\Delta L$ adjusted in the interferometer is known with a precision of $1 \mu \mathrm{m}$ and the thickness of the crystal $d$ is known with a precision of $10 \mu \mathrm{m}$, the group refractive index $N$ is obtained with a precision of $1 \times 10^{-4}$.

Then, we measured the group dispersion of the holey fiber in the setup shown in Fig. 1 in which the sample under test was replaced by components shown in Fig. 2(a) or 


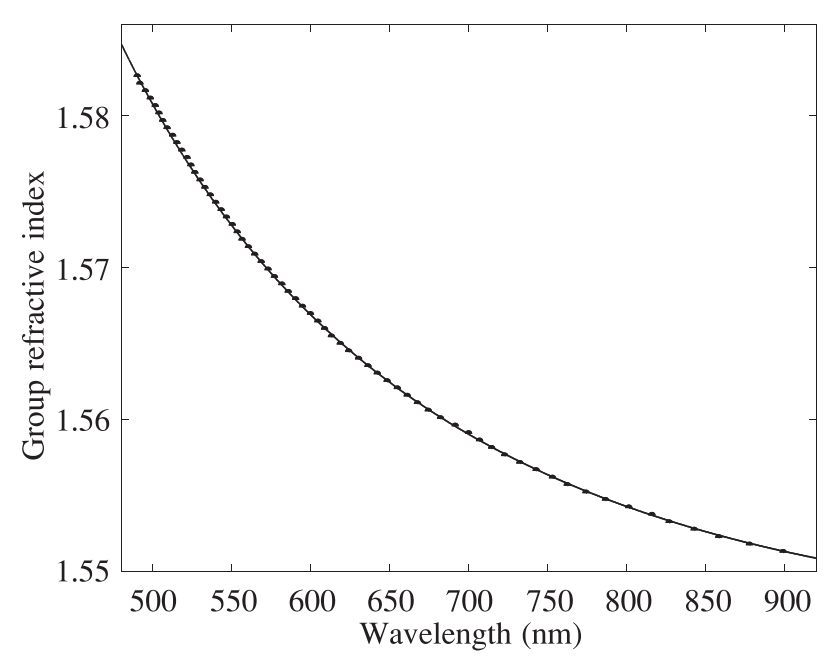

Fig. 4. The group refractive index of the quartz crystal as a function of the wavelength (solid line corresponds to theory).

2(b). In the first step we measured the group dispersion of components schematically shown in Fig. 2(b). Using the laser diode instead of the halogen lamp, we checked by observing the interference fringes that the components were placed precisely in the test arm. It should be noted here that the alignment of the components is much simpler in the interferometer with a single pass of light through the test arm than in a Michelson interferometer with two passes. Figure 5 shows an example of the spectral signal recorded for the path length difference $\Delta L=7182 \mu$ m with the equalization wavelength $\lambda_{0}=601.35 \mathrm{~nm}$. In this case the method of tandem interferometry was used because the path length difference $\Delta L$ corresponding to the components is smaller than the minimum path length difference $\Delta L_{\min }$ adjusted in the Mach-Zehnder interferometer. Figure 6 then shows by the dots the path length difference $\Delta L_{\min }\left(\lambda_{0}\right)$ as a function of the equalization wavelength $\lambda_{0}$ measured with a constant step of $20 \mu \mathrm{m}$. Solid line is the polynomial fit.

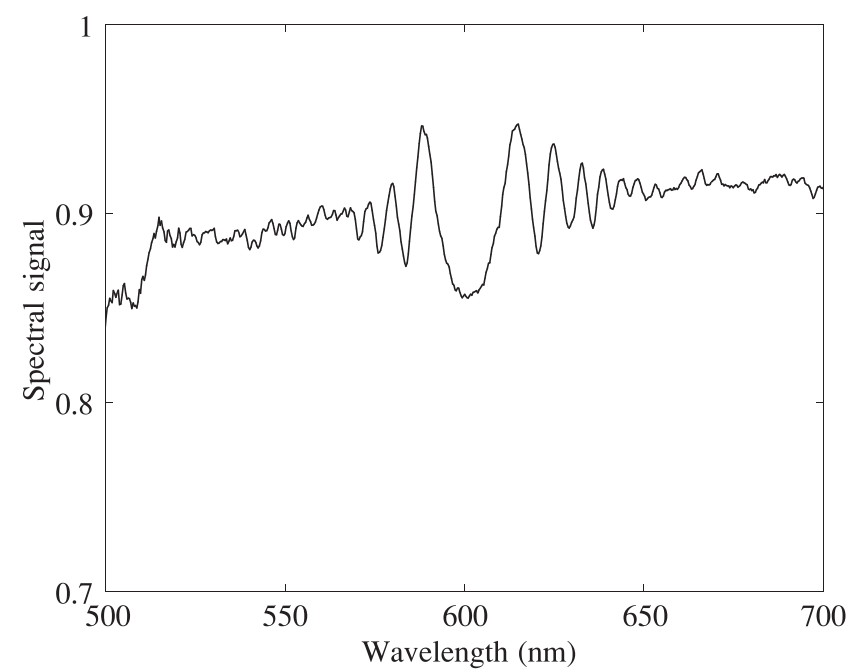

Fig. 5. Example of the spectral signal recorded for the path length difference $\Delta L=7182 \mu \mathrm{m}$ (microscope objective and achromatic lens).

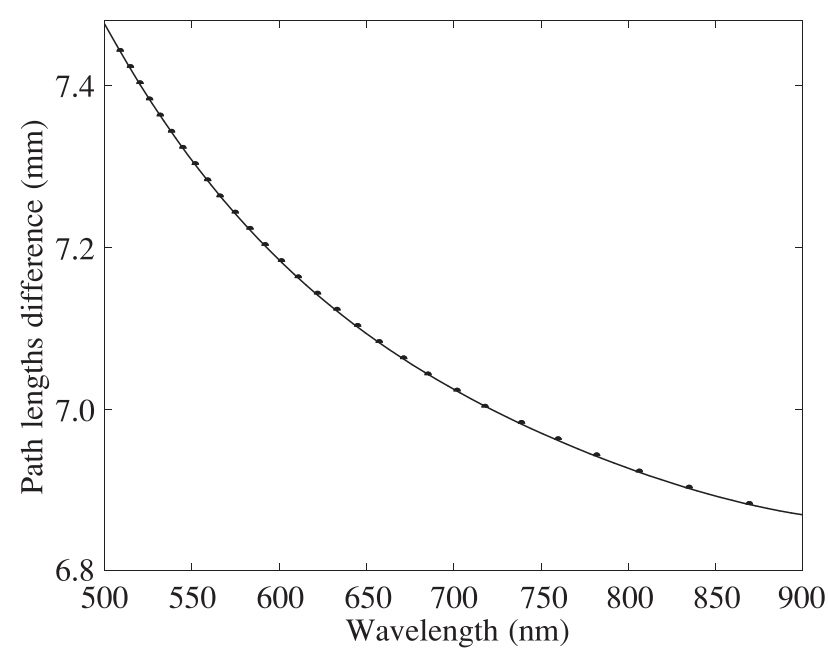

Fig. 6. The path length difference as a function of the wavelength for the combination of the microscope objective and achromatic lens (solid line is polynomial fit).

Finally, Fig. 7 shows an example of the spectral signal recorded for the components shown in Fig. 2(a) for the path length difference $\Delta L^{\prime}=31092 \mu \mathrm{m}$ to which the equalization wavelength $\lambda_{0}=656.07 \mathrm{~nm}$ corresponds. Subtracting the path length difference corresponding to two components shown in Fig. 2(b) we obtain $\Delta L=24004 \mu \mathrm{m}$ for the fiber alone. We revealed that the equalization wavelength $\lambda_{0}$ can be resolved in the spectral range from 505 to $912 \mathrm{~nm}$ and that the path length difference $\Delta L$ varies from 24793 to $23526 \mu \mathrm{m}$. The corresponding differential group refractive index $\Delta N\left(\lambda_{0}\right)=N\left(\lambda_{0}\right)-N\left(\lambda_{0 r}\right)$ as a function of equalization wavelength $\lambda_{0}$ is shown in Fig. 8 by the dots when $\lambda_{0 r}=748.54 \mathrm{~nm}$ was chosen. The values were calculated from the measured path length differences $\Delta L$ and the known length $z$ of the fiber [see Eq. (3)]. The solid line shows the same dependence for fused silica [8]. We estimate that the corresponding group refractive index can be obtained with a precision of $1 \times 10^{-4}$.

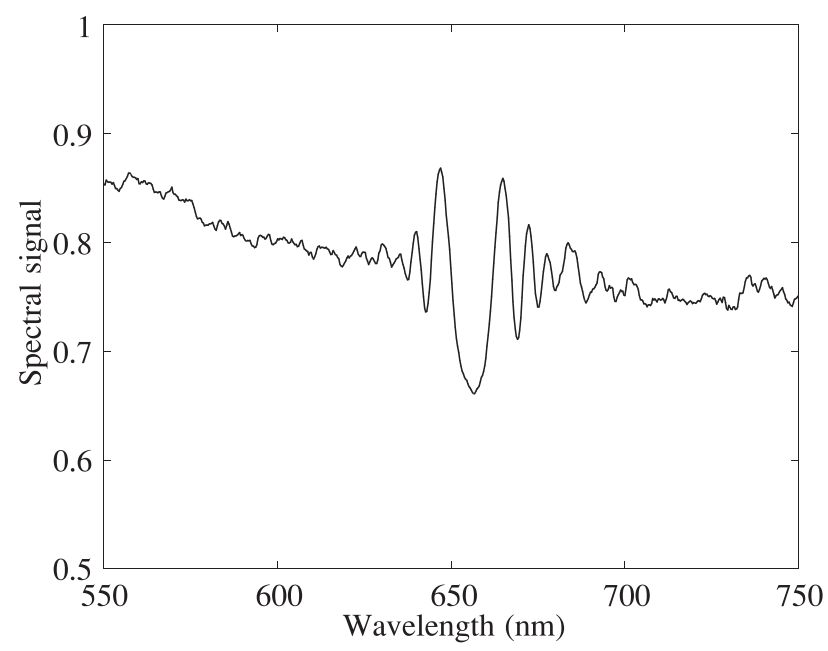

Fig. 7. Example of the spectral signal recorded for the path length difference $\Delta L=24004 \mu \mathrm{m}$ (holey fiber). 


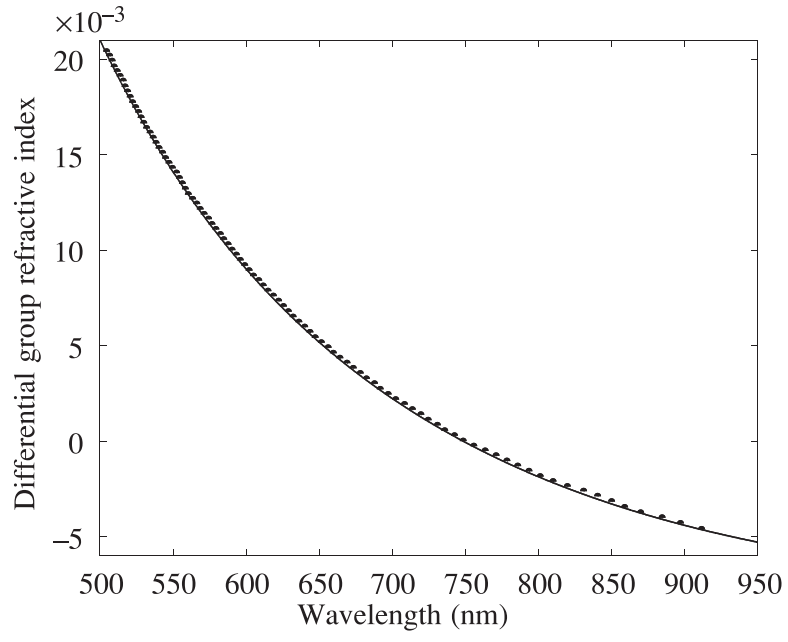

Fig. 8. The differential group refractive index of the fiber as a function of the wavelength (solid line corresponds to fused silica).

\section{Conclusions}

We used a simple white-light spectral interferometric technique for measuring the group dispersion of optical components over a wide wavelength range. The technique utilizes an unbalanced Mach-Zehnder interferometer with a component under test inserted in one arm and the other arm with adjustable path length. We recorded a series of spectral interferograms to measure the equalization wavelength as a function of the path length difference. We measured the group refractive index as a function of wavelength for a quartz crystal of known thickness. We measured also the relative group refractive index dispersion for optical fiber. In this case we used a microscope objective in front and a lens behind the fiber and subtracted their group dispersion, which was measured by a technique of tandem interferometry employing also a Michelson interferometer. The technique is applicable to any dispersive component with arbitrarily high dispersion or thickness, provided that the spectral interference fringes are resolved.

\section{Acknowlegments}

The research was partially supported by the Grant Agency of the Czech Republic (projects 102/06/0284, 202/06/ 0531), by the grant MSM6198910016, by an internal grant of TU Ostrava (IGS HGF VSB-TUO), and by an MŠMT grant (OC142) within the COST Action P11.

\section{References}

1. W.H. Knox, N.M. Pearson, K.D. Li, and C.A. Hirlimann, "Interferometric measurements of femtosecond group delay in optical components", Opt. Lett. 13, 574-576 (1988).

2. S. Diddams and J.C. Diels, "Dispersion measurements with white-light interferometry", J. Opt. Soc. B13, 1120-1128 (1995).

3. M. Galli, F. Marabelli, and G. Gizzetti, "Direct measurement of refractive-index dispersion of transparent media by white-light interferometry", Appl. Opt. 42, 3910-3914 (2003).

4. C. Sainz, P. Jourdain, R. Escalona, and J. Calatroni, "Real time interferometric measurements of dispersion curves", Opt. Commun. 110, 381-390 (1994).

5. V.N. Kumar and D.N. Rao, "Using interference in the frequency domain for precise determination of the thickness and refractive indices of normal dispersive materials", $J$. $\overline{O p t}$. Soc. B12, $\overline{1559-1563} \overline{(1995)}$.

6. Y. Liang and C.H. Grover, "Modified white-light MachZehnder interferometer for direct group-delay measurements", Appl. Opt. 37, 4105-4111 (1998).

7. H. Delbarre, C. Przygodzki, M. Tassou, and D. Boucher, "High-precision index measurement in anisotropic crystals using white-light spectral interferometry", Appl. Phys. B70, 45-51 (2000).

8. P. Hlubina, "White-light spectral interferometry with the uncompensated Michelson interferometer and the group refractive index dispersion in fused silica", Opt. Commun. 193, 1-7 (2001).

9. P. Hlubina and W. Urbanczyk, "Dispersion of the group birefringence of a calcite crystal measured by white-light spectral interferometry", Meas. Sci. Technol. 16, 1267-1271 (2005).

10. P. Hlubina and I. Gurov, "Spectral interferograms including the equalization wavelengths processed by autoconvolution method", Proc. SPIE 5064, 198-205 (2003).

11. G. Ghosh, "Dispersion-equation coefficients for the refractive index and birefringence of calcite and quartz crystal", Opt. Commun. 163, 95-102 (1999).

12. P. Hlubina, D. Ciprian, and L. Knyblová, "Direct measurement of dispersion of the group refractive indices of quartz crystal by white-light spectral interferometry", Opt. Commun. 269, 8-13 (2007). 